\title{
Gramática y gramáticas: entre el formalismo y el funcionalismo
}

\author{
Grammar and grammars: between formalism and \\ functionalism
}

Álvaro William Santiago Galvis ${ }^{1}$

Resumen

En este trabajo se presentan las distintas nociones que tiene el término gramática, desde la perspectiva prescriptiva, referida al sistema organizativo de la lengua, reglas y principios que intervienen en la construcción de palabras, frases y oraciones; pasando por la no prescriptiva, de orientación científica, que estudia sistemáticamente las palabras, sus clases, posibles combinaciones, relaciones y significados que puedan atribuírseles; hasta llegar a la relacionada con los enfoques lingüísticos específicos, o cada teoría o modelo de análisis que se ha propuesto para abordar un sistema verbal. Es a partir de la inmersión en esta última acepción que se estudian los presupuestos básicos de los programas formalista y funcionalista. El primero de ellos es utilizado por la gramática como un aparato formal de descripción y explicación con el que se busca aclarar la capacidad de la mente humana para producir y comprender expresiones verbales, sin tener en cuenta factores no verbales, mientras que el segundo hace énfasis en la función comunicativa de las lenguas, de forma que la gramática queda subordinada a factores extralingüísticos.

\section{Palabras clave}

Gramática, lenguaje, lengua, formalismo, funcionalismo.

\section{Summary}

In this work the different concepts that the word grammar has are presented, from the prescriptive perspective, referred to the organizational system of the language, rules and principles involved in the construction of words, phrases and sentences; passing through non-prescriptive perspective, which has a scientific orientation that studies the words systematically, their kinds, combinations, relations and meanings that may be assigned to them; up to that related to specific linguistic approaches, or every theory or model of analysis that has been proposed to address a verbal system. It is from this latter conception that the basic assumptions of formalist and functionalist programs are. The first one as a formal unit of description and explanation in grammar which seeks to clarify the human mind's ability to produce and understand utterances, regardless of nonverbal factors, while the latter emphasizes in the communicative function of language, so that grammar is subject to extralinguistic factors.

\section{Keywords}

Grammar, language, tongue, formalism, functionalism.

Artículo recibido el 30 de septiembre de 2010 y aprobado 28 de marzo de 2011

1 Profesor Departamento de Lenguas, Universidad Pedagógica Nacional; miembro del Grupo de Investigación en Pedagogía, Lenguaje y Comunicación, GIPELEC. asantiago@pedagogica.edu.co 
Guiraud, en su trabajo La gramática (1964), plantea que ésta se suele definir como el arte de hablar y escribir correctamente, en este sentido, la gramática se asume como "el conjunto de reglas que gozan de autoridad en un habla dada, en virtud de una norma establecida por los gramáticos o aceptada por el uso" (p. 7). Esta noción se puede considerar como producto de lo que en la historia de los estudios lingüísticos que se conoce bajo el rótulo de gramática tradicional; orientación ésta que se remonta a los estudios que, desde el siglo III a.C., adelantaran los griegos y los hindúes sobre los textos arcaicos (los poemas homéricos y los himnos vedas). Esta tradición, entre otros aspectos, se sustentaba en una visión prescriptiva de la lengua, esto es, ofrecer un conjunto de normas para expresarse, ya sea por medio del código verbal o del escrito, de forma correcta $^{2}$. Visión que llegará a nuestros días ${ }^{3}$ gracias a lo que se llama la escuela greco-latina, de la cual, como plantea Hjelsmlev, occidente es heredera.

Según Guiraud, esta manera de entender el término gramática corresponde a una visión normativa, que difiere de la lingüística o ciencia de las lenguas en lo que tiene que ver con sus principios, estructuras y relaciones. De esta forma, se plantea que, con el fin de superar el tinte normativo de la gramática tradicional, se asuma mejor el término de lingüística, dado el carácter de ciencia que esta noción comporta, lo cual está ligado al hecho de que Saussure, al establecer los fundamentos de la lingüística, asume que el objeto de estudio de ésta ha de ser la lengua; así, se plantea, y se configurará con el estructuralismo, una lingüística de la lengua, en la que la gramática dejará de lado el carácter prescriptivo para adquirir una orientación científica, o por lo menos con esta intención, que, en principio,

2 No obstante, como señala Marcos (1994, p. 29), los griegos ofrecen junto al enfoque normativo, otra orientación con respecto al estudio de la lengua, que se caracteriza por ser de tipo teórico, lógico-filosófico y especulativo; esta orientación se “preocupa por la conexión del lenguaje con el pensamiento, por las categorías universales, las partes de la gramática", entre otros aspectos.

3 De hecho, en su vigésima primera edición, el DRAE define la gramática como: "Arte de hablar y escribir correctamente una lengua", y esta noción se presenta como la primera acepción del término. En su vigésima segunda edición, el DRAE presenta esta definición, pero ya no como la primera acepción sino como la cuarta. se ocupará del análisis de las formas y las construcciones (Guiraud, p. 8).

De acuerdo con lo anterior, tenemos dos maneras de entender la noción de gramática: una de corte normativo y otra de orientación científica ${ }^{4}$. Una centra su interés en la prescripción del buen decir, mientras que la otra aspira a ser un estudio sistemático de los elementos constitutivos de la lengua, sean éstos sonidos, formas, palabras o construcciones. Aspiración completamente válida, pues como señalara Hjelsmlev, en los albores de la centuria pasada, "la gramática puede y debe ser una disciplina científica” (1976, p. 24).

Esto lleva a plantear que gramática es, al igual que otras nociones propias de la lingüística, el lenguaje ${ }^{5}$ por ejemplo, un término que tiene varios sentidos, los cuales difieren, según Alcaraz y Martínez (2004), tanto en su extensión como en la naturaleza del objeto al que se refieren. De acuerdo con estos autores, desde el objeto de estudio, se pueden plantear dos grandes significados para el término gramática:

1) Se emplea para hacer referencia a un aspecto particular de un sistema verbal, el cual corresponde al "sistema por el que las palabras y morfemas de una lengua se organizan en unidades mayores, particularmente oraciones, percibido como existente con independencia de cualquier intento de describirlo" (Trask, citado por Alcaraz y Martínez, 2004, p. 319). Así, la gramática se refiere al sistema organizativo de la lengua, es decir, "el conjunto de principios y reglas que actúan en la construcción de palabras, frases y oraciones ${ }^{26}$ (Fages, 2005, p. 22).

4 Al respecto, Klinkenberg (2006, p. 120) plantea que la palabra gramática tiene dos sentidos: uno de carácter prescriptivo y otro de tipo no prescriptivo. El primero tiene que ver con "una lista de prescripciones normativas, del género 'di, no digas' , o mejor dicho una serie de indicaciones prácticas". En el sentido no prescriptivo, gramática "designa a la vez (a) un dispositivo productor de enunciados, y $(b)$ la descripción de dicho dispositivo".

5 Este aspecto, precisamente, se suele presentar como una de las debilidades de la lingüística como ciencia, en el sentido de que sus conceptos básicos, lenguaje y lengua, presentan problemas de caracterización y delimitación, amén de los problemas metodológicos.

6 Aquí se hace necesario señalar que esta caracterización corresponde a gramáticas de corte oracional, esto es, aquellas que tienen como unidad de análisis la oración; no obstante hay orientaciones 
2) Hace alusión a una disciplina científica determinada, esto es, la disciplina lingüística que tiene como objeto el análisis de ese aspecto particular de un sistema verbal. De esta forma, la gramática se entiende como "la disciplina que estudia sistemáticamente las clases de palabras, las combinaciones posibles entre ellas y las relaciones entre esas expresiones y los significados que puedan atribuírseles" (Bosque y Demonte, 1999, pág. XIX).

Frente a estas dos nociones se puede plantear una tercera, en la que se entiende la gramática como el conocimiento que tiene una persona que habla determinada lengua; este conocimiento constituye lo que, desde la perspectiva chomskiana, se conoce como la competencia lingüística del hablante, conocimiento que podemos caracterizar como implícito y que está relacionado con ese sistema organizativo de la lengua.

Ahora bien, en cuanto a la segunda acepción, Alcaraz y Martínez plantean que cuando con la noción de gramática se hace alusión a una disciplina lingüística, ésta también tiene, al menos, dos significados, según la extensión o el alcance del estudio:

1) Un significado restringido, de vieja data, que tiene que ver con la disciplina que se ocupa básicamente de la sintaxis y la morfología, esto es, la gramática se encarga de estudiar la combinación de las palabras para conformar oraciones y, de otra parte, la articulación de los morfemas para configurar unidades léxicas. Ésta es, entonces, una concepción eminentemente formal, que deja por fuera de su ámbito de trabajo la fonética y la semántica. De acuerdo con esta visión, la gramática tiene dos unidades de análisis: una mínima, correspondiente al morfema, y otra máxima, la oración que, a la vez, se constituye en el elemento central del trabajo gramatical. La combinación de estas dos unidades lleva a que se asuma la morfosintaxis como sinónimo de gramática. Esta disciplina tiene como objetivo adelantar "el

supraoracionales, las cuales toman como unidad de análisis el texto o discurso, lo que ha llevado a que se tenga una gramática del texto que asume que éste tiene una estructura o configuración particular, en consecuencia, la forma de analizarlo es también particular. análisis y descripción, en sus aspectos formales $\mathrm{y}$ funcionales, de las unidades significativas que componen los enunciados, así como el análisis y descripción de los recursos sistemáticos que permiten combinarlos para formar los enunciados" (Alcaraz y Martínez, p. 319).

2) Un significado amplio: este tiene que ver en gran medida, aunque no de forma exclusiva, con la llamada teoría generativa, dado que para ésta la gramática viene a ser equivalente a "teoría de la lengua", la cual incluye tanto la fonología como la semántica y toma la sintaxis como el componente central, por lo que se inscribe, en principio, dentro de un enfoque formalista.

Para Alcaraz y Martínez, otra acepción del término gramática se da cuando se emplea con especificaciones como estructural, de dependencias, de casos, de construcción, formal, funcional, generativa, cognitiva, comparada, histórica, descriptiva. En este caso, el término gramática se usa para hacer referencia a un enfoque lingüístico determinado, el cual está basado en una teoría particular. Bajo esta óptica, las gramáticas se tornan en sistemas teóricos que, en principio, comparten y coinciden en los datos que analizan, pero que difieren en los aspectos que consideran relevantes, "en las categorías que identifican y en los criterios utilizados para definirlas. Reflejan, por tanto, de forma distinta un mismo objeto y explican de manera diferente los hechos" (Alcaraz y Martínez, p. 319).

Teniendo en cuenta lo expuesto hasta ahora, podemos considerar que la polisemia del término gramática es posible sintetizarla como sigue:

1. El arte de hablar y escribir correctamente, que corresponde a la visión normativa.

2. Estudio sistemático de los elementos constitutivos de un sistema verbal: formas, palabras y construcciones; ésta acepción corresponde la visión científica.

3. Configuración estructural de cualquier código verbal, noción asociada al sistema como tal. 
4. Conocimiento que el hablante tiene de su lengua materna, esto es, su competencia lingüística, concepto relacionado con la concepción generativista.

5. Enfoques lingüísticos, es decir, cada uno de las teorías o modelos de análisis que se han propuesto para abordar un sistema verbal ${ }^{7}$.

De acuerdo con lo establecido en el numeral 5, y como señalan Bosque y Demonte (1999), las propiedades de los elementos de una lengua, así como las relaciones y combinaciones que se dan entre ellos, se pueden formular de diversas maneras, según el punto de vista teórico desde el que se aborde el estudio, lo cual lleva a que se tengan diversos enfoques gramaticales (o modelos lingüísticos, si se quiere), esto es, puede "haber muchas gramáticas de la Gramática de una lengua" (Bosque y Demonte, p. XIX) ${ }^{8}$, que atienden a determinar, grosso modo, cómo debe ser la lengua (perspectiva normativa), cómo se presenta (enfoque descriptivo), cómo funciona (óptica funcionalista), cómo se conoce (enfoque generativista), cómo conceptualiza el mundo (visón cognitiva) (cfr. López, 2000).

Así pues, estas formas de encarar el estudio de la lengua están en consonancia con una teoría lingüística, esto es, una forma de concebir el fenómeno lingüístico, y en consecuencia, de abordarlo conceptual y metodológicamente. Dichas orientaciones pueden llegar a diferir diametralmente en sus propuestas epistemológicas y metodológicas. Tal es el caso que se dio, por ejemplo, con la concepción generativista con respecto a la estructuralista, o la que en periodos más recientes se ha dado entre el programa formalista (o generativista) y el funcionalista.

La controversia entre estas dos últimas orientaciones sobre el estudio de la lengua supone,

7 Así las cosas, tenemos que la primera noción se ubica dentro de la visión prescriptiva, mientras que las restantes se relacionan con la concepción no prescriptiva.

8 Según esto, podemos tener una gramática normativa, una descriptiva, una estructural, una funcional, una generativa, una de dependencias, una léxico-funcional, una histórica, para la lengua española. Así, por ejemplo, se tiene: la Gramática Descriptiva de la Lengua Española, coordinada por Bosque y Demonte; la Gramática Transformativa del Español de Hadlich; la Gramática Estructura/de Alarcos. en términos de Mendívil (2003), tanto aspectos metodológicos como epistemológicos; uno de éstos, la naturaleza misma del objeto de estudio: la gramática. Mientras que para el generativismo, la gramática es un sistema de conocimiento autónomo, el funcionalismo rechaza este planteamiento.

En relación con la naturaleza del objeto, éste se puede ampliar y orientar la indagación hacia la naturaleza misma del lenguaje. Al respecto, como señala Lorenzo (2006), hay dos formas de ver el fenómeno lingüístico: la biológica y la socio-cultural. Esto es, hay una orientación teórica que asume el lenguaje como un rasgo biológico propio de la especie humana y, por ello, un aspecto más del mundo natural, la cual corresponde a lo que se ha denominado el giro biolingüístico ${ }^{9}$; desde esta perspectiva, se plantea que parece que existen genes que controlan el desarrollo de las estructuras cerebrales que tienen a su cargo la producción y la comprensión verbal, "estructuras que a su vez determinan de algún modo las propiedades de las gramáticas a través de las cuales se manifiestan tales habilidades" (Lorenzo, p. 16). Por su parte, la concepción sociocultural asume el lenguaje como un hecho de carácter cultural; en esta concepción se destaca el valor de la lengua como instrumento de comunicación, como sustento de las relaciones sociales, como depositaria de la tradición cultural de los pueblos.

Así, es posible plantear que existe una lingüística centrada en la dimensión biológica y otra en el aspecto o dimensión sociocultural. Esto está relacionado con el hecho de que el lenguaje tiene una naturaleza compleja que involucra aspectos biológicos (anatómicos, psicológicos, fisiológicos, neurológicos, genéticos) y sociales (culturales, sociales, simbólicos), los cuales difícilmente se pueden abordar de forma completa desde una orientación particular, sea ésta formalista o funcionalista, de

9 Este enfoque corresponde a lo que se ha denominado el giro biolingüístico. Éste plantea la posibilidad de caracterizar y ubicar a la lingüística -o una fracción de ella- como parte de los estudios biológicos, pretensión que se sustenta en la asunción del lenguaje como aspecto diferenciado de la constitución orgánica del ser humano; con base en ello se puede "considerar con todas las consecuencias a la Lingüística como el nombre propio de una rama (altamente especializada) de las ciencias de la vida" (Lorenzo, p. 11). 
ahí que se esté optando ahora por la denominación de ciencias del lenguaje como el conjunto de disciplinas que se ocupan, o sustentan su objeto de estudio, sobre algún aspecto del lenguaje, entre ellas, la gramática.

Ahora bien, en términos generales, para el generativismo, el objetivo de la investigación consiste en caracterizar las relaciones formales ${ }^{10}$ que se dan entre los elementos de la lengua, sin tener en cuenta aspectos relacionados con las propiedades semánticas o pragmáticas de aquellos. Esto lleva a plantear la autonomía de la gramática, más exactamente, de la sintaxis, de ahí que se asuma que el componente generador sea el sintáctico. De esta forma, las expresiones verbales son producto de la interacción de una serie autónoma de reglas y principios gramaticales.

El funcionalismo, entre tanto, no acepta este planteamiento bajo el argumento de que la "función básica de trasladar o comunicar significado es la responsable de la forma y estructura de la gramática, y que, por tanto, es imposible separarla realmente del valor simbólico del lenguaje" (Mendívil, p. 49). Esto lleva a que se considere que aspectos como la situación comunicativa determinan la estructura gramatical de las expresiones verbales y que la sola descripción formal no puede dar cuenta de estos elementos. En este sentido, se establece una relación entre las estructuras gramaticales y las situaciones comunicativas.

Al respecto se plantea que la gramática no sólo sirve para producir oraciones, ya sean afirmativas, interrogativas, negativas o dubitativas, sino para adelantar procesos de interacción verbal. Así se aboga por el estudio de la lengua en situación, los usos que los hablantes hacen del código verbal en situaciones comunicativas concretas, de tal forma que se busca relacionar la forma gramatical con "las necesidades, propósitos, medios y circunstancias de

10 El sustento de esta caracterización se da en la aplicación de formalismos inspirados en la matemática. Según esto, “las gramáticas son planteadas como axiomas, como máquinas teóricas o como constructos abstractos, capaces de generar y de interpretar las expresiones de una lengua como lo hacen los hablantes" (Cabrè y Lorente, 2003, p.3), recurriendo para ello a metalenguajes basados en la lógica formal y la matemática moderna. la comunicación humana" (Cabrè y Lorente, 2003, p. 10).

De acuerdo con Chomsky (1989) ${ }^{11}$, el acercamiento al fenómeno lingüístico, desde la perspectiva de la gramática generativa, ha entrañado un cambio en el planteamiento mismo de los problemas sobre la lengua y el lenguaje, pues propició un cambio radical en los estudios lingüísticos, al pasar de considerar simplemente el comportamiento o los productos del comportamiento lingüístico, a considerar los estados de la mente/cerebro que intervienen en dicho comportamiento; este cambio de perspectiva en el quehacer lingüístico entrañó el planteamiento de los siguientes interrogantes fundamentales ${ }^{12}$ para la investigación lingüística, según la visión generativista:

1. ¿En qué consiste el conocimiento de la lengua?

2. ¿Cómo se adquiere la lengua?

3. ¿Cómo se usa ese conocimiento?

La pregunta acerca del conocimiento de la lengua, esto es, qué elementos hay en la mente/cerebro del individuo que le permiten comprender y producir emisiones lingüísticas, se puede contestar de dos formas. En primer lugar, conocer una lengua se podría asumir como una habilidad práctica para entender y hablar; sin embargo, esta concepción no puede dar cuenta de cuál es la naturaleza misma de dicha capacidad y, a la vez, cómo se describe (analiza) y se adquiere.

Frente a esta limitación teórica, se argumenta que el conocimiento de la lengua consiste en cierto estado de la mente/cerebro del individuo; dicho estado es un elemento relativamente estable entre los estados mentales transitorios del individuo. Supone esta hipótesis que se trataría de un estado de una facultad mental diferenciable, que Chomsky no duda en llamar facultad del lenguaje, la cual se carac-

11 La exposición que se hace de los postulados del formalismo y del funcionalismo está, en parte, basada en el artículo La lengua: su estudio y su enseñanza, que publicamos en el año 2007 en la revista Praxis Pedagógica, No. 8.

12 Junto a estos interrogantes, se encuentran: ¿Cuáles son los mecanismos físicos que sirven de base a dicho sistema de conocimiento y al uso del mismo? y ¿cómo evoluciona este conocimiento en la especie? Estas dos preguntas abren las puertas para la investigación en biolingüística y neurolingüístca. 
terizaría por tener ciertas propiedades, así como una estructura y una organización específicas. Desde esta perspectiva, la facultad del lenguaje correspondería a un módulo de la mente, esto según la teoría que sostiene que la mente/cerebro humana es un sistema complejo, conformado por varios componentes que interactúan,

a uno de los cuales podemos llamarle "facultad del lenguaje". Este sistema parece ser exclusivo de la especie humana, en lo esencial y común a los miembros de la especie. Una vez provista de datos, la facultad del lenguaje determina una lengua particular, el español, el inglés, etc. Esta lengua a su vez determina un amplio espectro de fenómenos potenciales que va mucho más allá de los datos recibidos (Chomsky, 1988, p. 37).

Según ello, cuando el individuo posee una lengua, desarrolla un sistema particular de conocimiento, representado de alguna forma en la mente y ubicado físicamente en algún lugar del cerebro, en una especie de configuración física (Chomsky, 1988); en este sentido, la lengua se ve como una realidad cognitiva (sistema de conocimiento de la mente) y física (debe poseer un sustrato fisiológico en el cerebro). De esta forma, para el generativismo, la lengua es un tecnicismo con el cual se hace referencia a un sistema de conocimiento interiorizado en la mente del individuo; en este sentido, la lengua es un hecho de la psicología individual, lo que entra en oposición con el carácter social de los enfoques funcionalistas.

Esto lleva a que se diferencie teoría lingüística de gramática. Así, la teoría lingüística corresponde al "sistema de hipótesis acerca de los rasgos generales del lenguaje humano, propuesto para dar cuenta de un conjunto determinado de fenómenos lingüísticos" (Chomsky, citado por Fernández y Anula, 1995, p. 32). Estos fenómenos lingüísticos son las lenguas, de esta forma, la teoría lingüística centra su interés en determinar y establecer las propiedades universales de las lenguas. Por su parte, la gramática se entiende, desde esta perspectiva, como la descripción de la competencia lingüística del hablanteoyente ideal. Esto implica, según Fernández y Anula, que a nivel teórico se reconoce la existencia de un hablante-oyente idealizado y que éste posee un tipo particular de conocimiento: la lengua-I, que se entiende como "algo en la mente de la persona que conoce el lenguaje, algo que adquiere el que aprende el lenguaje y que usa el oyente-hablante" (Chomsky, 1989, p. 171). Esta lengua-I se convierte en el objeto de la investigación gramatical.

De otro lado, el generativismo plantea dos conceptos diferenciados de gramática: la gramática universal (GU) y la gramática particular (GP). La gramática universal corresponde al estado inicial (Ei) de la facultad del lenguaje; esta gramática es "común a la especie humana y cuyos principios son semejantes en todas las lenguas" (Fernández y Anula, 1995, p. 32). Esto en consonancia con el planteamiento de que la facultad del lenguaje es un sistema diferenciado de la mente/cerebro que presenta un estado inicial (Ei), "común a la especie y aparentemente exclusiva de la especie en ciertos aspectos esenciales" (Chomsky, 1989, p. 173). Esta gramática se constituye en el objeto de estudio de la teoría lingüística, la cual "se refiere a la teoría de los lingüistas sobre el estado inicial de la facultad del lenguaje, el componente último de la mente/ cerebro, la parte fijada por la dotación biológica" (Fernández y Anula, p. 33).

La gramática particular, entre tanto, hace referencia a la organización de los distintos sistemas verbales, los cuales son posibles gracias a los principios de la gramática universal. Las gramáticas particulares, por lo tanto, son teorías sobre las distintas lenguas, "más concretamente, son teorías acerca del componente generativo de la facultad lingüística denominado lengua-I, encargado de generar las expresiones del lenguaje" (Fernández y Anula, p. 32). Así, la lengua-I se puede entender como una realidad u objeto físico que se encuentra en la mente/ cerebro de los individuos, de ahí su carácter de interna; esta realidad es individual y no de carácter social, por ello se plantea que es un hecho de la psicología individual; y cuenta con un mecanismo generativo finito que ofrece las descripciones estructurales del lenguaje. Según esto, la gramática tiene que ver con la teoría de los lingüistas sobre la lengua-I. 
La introducción de la noción de lengua-I o lengua internalizada surge como oposición a la de lengua externalizada (lengua-E), que no es otra cosa que el conjunto de oraciones con el que se suele definir el lenguaje; esta lengua-E es externa a la mente/cerebro dado que es producto de la capacidad, mas no es la capacidad; por su parte, como hemos señalado, el concepto de lengua-I corresponde a la noción de estructura mental del individuo que le orienta para crear sus emisiones lingüísticas. Este planteamiento lleva a establecer que:

a. El lenguaje corresponde en esencia a la lengua-I.

b. La gramática o el estudio de la lengua es, ante todo, una teoría de la lengua-I; esta se constituye, en consecuencia, en su verdadero objeto de estudio.

c. Conocer una lengua $\mathrm{L}$ es una propiedad o, mejor, un estado de la mente/cerebro de una persona $\mathrm{P}$, de tal forma que se plantea que "para $\mathrm{P}$ conocer el lenguaje L consiste en que la mente/cerebro de P está en un cierto estado y más estrictamente, que la facultad del lenguaje, un módulo de ese sistema, está en cierto estado SL" (Chomsky, p. 171).

d. De acuerdo con lo anterior, proponer que $\mathrm{P}$ conoce $\mathrm{L}$ "equivale a decir que $\mathrm{P}$ tiene cierto lenguaje-I", esto implica formular la hipótesis de que la Gramática Universal se constituye en la teoría general de las características y propiedades de los lenguajes-I humanos. Así, esta teoría centra su atención en determinar "las condiciones derivadas de la dotación biológica humana que identifica los lenguajes-I humanamente accesibles en condiciones normales" (Chomsky, p. 172).

Siguiendo con la argumentación de Chomsky, la gramática generativa cambió el interés de la lingüística de la lengua-E a la lengua-I, lo cual condujo a poner en evaluación el planteamiento de que la facultad del lenguaje es un sistema diferenciado de la mente/ cerebro que presenta un estado inicial (E0), el cual, en contacto con los datos adecuados, pasa a un estado relativamente estable E1, que corresponde a la lengua-I, y esta posibilita la producción de las emisiones lingüísticas (L-E). El estado inicial es el objeto de la Gramática Universal, mientras que los estados estables se constituyen en el objeto de las gramáticas particulares, propias de cada lengua. No obstante, los dos son objeto de estudio de la gramática generativa.

Frente a esta visión formalista del fenómeno lingüístico, que Halliday llama la perspectiva intra-organismos, dado que su objetivo es el de caracterizar cuál es el sistema de conocimiento que posee el individuo en su mente/cerebro que le permite producir y comprender oraciones, se erige la visión inter-organismos, la cual asume el lenguaje como comportamiento humano que se da entre individuos. En palabras de Halliday (1982, p. 32), esta perspectiva plantea que un hecho importante respecto del habla y la comprensión de la lengua radica en que siempre se produce en un contexto. No sólo "conocemos" nuestra lengua materna como un sistema abstracto de signos vocales, o como si fuera una especie de texto de gramática con un diccionario adjunto; la conocemos en el sentido de saber cómo utilizarla, sabemos cómo comunicarnos con otras personas, cómo elegir formas de lenguaje apropiadas al tipo de situación en que nos encontramos, y así sucesivamente". En relación con este planteamiento, cabe señalar que la noción de competencia comunicativa, planteada por Hymes desde la sociolingüística, hace énfasis en el hecho de que conocer una lengua no es sólo conocer su estructura gramatical, sino que implica otra serie de aspectos que llevan a que el individuo pueda interactuar de manera adecuada en una situación comunicativa particular.

La aproximación funcionalista plantea que la lengua juega un papel, en el sentido de cumplir una función ${ }^{13}$, básico en el proceso de socialización del

13 En términos de funciones del lenguaje, para Chomsky, la función fundamental consiste en la representación, es decir, el lenguaje sirve "para representar la realidad 0 , en otros términos, para organizar coherentemente lo que percibimos, conceptualizamos e imaginamos" (Bernárdez, 1999, p. 277). En este sentido, Chomsky argumenta que podemos comunicarnos de diversas formas, utilizando distintos sistemas sígnicos no verbales, pero sin el lenguaje no podemos representarnos la realidad de forma organizada, de ahí 
individuo; desde esta óptica, sólo por medio de la lengua el individuo puede integrarse al grupo, "a 'la gente', pero a su vez, la 'gente' está compuesta de 'personas'; en virtud de su participación en un grupo, el individuo ya no sólo es un espécimen biológico de humanidad: es una persona" (Halliday, p. 24). Lo anterior debido a que la lengua es elemento básico para la interacción social. Esto remite al planteamiento central del funcionalismo, que entra en oposición con el del generativismo: la lengua es, ante todo, un instrumento de comunicación social; en este sentido, según Dik (1981, p. 19), la lengua se entiende, fundamentalmente, "como un instrumento de interacción social entre los seres humanos, usada con el objetivo primordial de establecer relaciones de comunicación entre hablantes y destinatarios". De acuerdo con este teórico, la lingüística de corte funcional debe ocuparse de dos sistemas de reglas de origen social: a) las reglas que rigen la interacción verbal como una forma de actividad cooperativa (reglas pragmáticas) y b) las reglas que rigen las expresiones lingüísticas estructuradas utilizadas como instrumentos en esa actividad (reglas semánticas, sintácticas, morfológicas y fonológicas) (Dik, p. 20).

Así, para el paradigma funcional, desde la visión de Dik, una lengua es un instrumento de comunicación, de ahí que la función primaria de ésta sea la interacción entre los individuos; en este sentido, el correlato psicológico del sistema verbal es la competencia comunicativa, entendida como habilidad para mantener la interacción social por medio del código verbal. $\mathrm{Al}$ analizar la lengua, la pragmática es el marco globalizador dentro del cual debe estudiarse la semántica y la sintaxis; de esta forma, la semántica está subordinada a la pragmática y la sintaxis a la semántica, con lo cual se deja de lado el planteamiento de la autonomía de la sintaxis y se le otorga un papel central al componente semántico. Esta orientación busca dar cuenta del sistema de la lengua a través de los usos que se hacen de las expresiones lingüísticas en la interacción social. Según

que se caracterice al lenguaje como facultad que permite organizar y jerarquizar la realidad, lo cual se refleja en la organización de las gramáticas de las lenguas. esto, los enfoques funcionalistas se fundamentan en la hipótesis que sostiene que "el lenguaje está motivado pragmáticamente, o sea que las lenguas, tal como las conocemos, se han ido configurando como respuestas a las necesidades de comunicación en acciones interactivas ubicadas en contextos específicos" (Cabrè y Lorente, 2003, p. 13).

De acuerdo con Cabrè y Lorente, las escuelas de corte funcionalistas se orientan por los siguientes principios teóricos:

1. La función fundamental del lenguaje es la comunicación.

2. El lenguaje está motivado pragmáticamente, aspecto que condiciona el significado, esto es, las expresiones lingüísticas adquieren valor según las circunstancias en las que se usen.

3. El significado, que se encuentra restringido pragmáticamente, incide por lo tanto en la estructuración o configuración gramatical de los distintos sistemas verbales.

4. Los universales lingüísticos están relacionados con las funciones del lenguaje.

5. El lenguaje es un proceso dinámico.

6. El control externo de los actos comunicativos explica la variación lingüística.

La gramática de orientación funcionalista presenta una serie de orientaciones y enfoques, tal es el caso del funcionalismo europeo y del americano, no obstante todos comparten el hecho de concebir el lenguaje -la lengua- como instrumento de comunicación (principio 1). Así, por ejemplo, la Gramática del papel y la referencia, de Van Valin (cfr. González, 2006), se asume como una teoría que se inscribe en la visión funcionalista, dado que parte del principio de que las lenguas posibilitan la vida social gracias a que brindan la opción de la interacción verbal; en este sentido, la lengua es un sistema de comunicación y no un conjunto infinito de descripciones estructurales de oraciones, esto es, la lengua es una herramienta para la comunicación y no las descripciones formales que hacen los lingüistas.

En este modelo se tiene en cuenta aspectos semánticos y pragmáticos, en consecuencia, no se acepta la autonomía de la sintaxis y se postula la existencia de tres componentes en el sistema de 
representación lingüística: el semántico, el sintáctico y el informativo. Según este modelo, la oración se debe entender como una estructura gramatical que los hablantes utilizan para describir sucesos que ocurren en el mundo, ya sea éste real o posible; estos sucesos se entienden como estados de cosas, los cuales se pueden clasificar en no dinámicos (estados, condiciones, cualidades), eventos (cosas que suceden de forma instantánea), procesos (cambios a lo largo de un período) y acciones (describe algo que una entidad hace); lo anterior se puede clasificar en dos grandes categorías: estados y actividades. Estos elementos formarán parte de la estructura semántica, los cuales luego se formalizarán en la estructura sintáctica, a través de funciones sintácticas, para llegar finalmente a la realización fónica. En este punto cabe resaltar que hay planteamientos que señalan que la lengua es un sistema que media, de forma compleja, entre el universo del significado y el universo de los sonidos (cfr. Tobón, 2007). De ahí que la producción verbal se asuma como un proceso que va de la configuración conceptual -proposición- a la cadena fónica -enunciado-, por medio del cual el hablante hace uso del sistema verbal en situaciones comunicativas concretas.

Como hemos visto, el panorama de los estudios gramaticales es complejo y diverso. Ahora bien, esto es prueba de que estos estudios se han constituido en un capítulo importante de las ciencias del lenguaje, por no hablar de la lingüística. Los enfoques con respecto a la gramática van desde los estrictamente normativos hasta llegar a los pragmáticos, sin dejar de lado los pedagógicos y didácticos. Al respecto, se puede hablar de una gramática aplicada, la cual tiene entre sus campos de acción la enseñanza de las

\section{Bibliografía}

Alcaraz, E. y Martínez, M. (2004). Diccionario de lingüística moderna. Barcelona: Ariel.

Allen, J. (1987). Gramática pedagógica. En J. Álvarez, Teoría lingüistica y enseñanza de la lengua (147-171). Madrid: Akal.

Bernárdez, E. (1999). ¿Qué son las lenguas? Madrid: Alianza. lenguas -sean éstas materna, segunda o extranjera-, de ahí que surja la noción de gramática pedagógica, que busca "ofrecer un cuadro comparativamente informal de definiciones, esquemas, ejercicios y reglas explícitas que puedan ayudar al estudiante a adquirir un conocimiento del lenguaje y un uso fluido" (Allen, 1987, p. 148).

La importancia de los estudios sobre la lengua y la gramática, pese a las diferencias teóricas, se sustenta en el hecho de que existe un objeto real del mundo real, como señala Mendívil, que merece ser analizado y explicado con el rigor que supone el trabajo científico.

No obstante las diferencias -a veces radicales- de las propuestas planteadas en el siglo pasado y en lo que va corrido de éste, la gramática ha logrado consolidarse como disciplina científica, a tal punto que ha desarrollado un aparato formal de descripción y explicación bastante elaborado, tal es el caso del enfoque formalista, con el cual se busca dar cuenta de la capacidad de la mente/cerebro del individuo para producir y comprender expresiones verbales, sin tener en cuenta factores no verbales.

Frente a ello, los enfoques funcionalistas, sin dejar de lado el formalismo, en términos de generar algoritmos que sustenten su propuesta teórica, hacen énfasis en la función comunicativa de las lenguas, de tal forma que la gramática está supeditada a factores extralingüísticos, lo cual ha llevado a que se establezcan diferenciaciones, en los modelos lingüísticos, entre los no sensibles y los sensibles al contexto. Esta dicotomía, si la podemos llamar así, enriquece la comprensión de un fenómeno que, en su esencia, es tan complejo como el ser que lo ostenta.그

Bosque, I. y Demonte, V. (1999). Gramática descriptiva de la lengua española. Madrid: Espasa-Calpe.

Cabrè, T. y Lorente, M. (2003). Panorama de los paradigmas en lingüística. Recuperado julio 15 de 2010. Disponible en: www.textosenlinea.com.ar/.../ Panorama\%20de\%20los\%20paradigmas\%20en\%20 linguistica.pdf 
Chomsky, N. (1988). El lenguaje y los problemas del conocimiento. Madrid: Visor.

(1989). Cambios de la perspectiva sobre el conocimiento y uso del lenguaje. En A. AlonsoCortés (Ed.), Lecturas de lingüística (163-201). Madrid: Cátedra.

Dik, S. (1981). Gramática funcional. Madrid: Sociedad General Española de Librería.

Fages, X. (2005). Gramática para estudiantes. Barcelona: Laertes.

Fernández, M. y Anula, A. (1995). Sintaxis y cognición. Madrid: Síntesis.

González, C. (2006). La Gramática del papel y la referencia: una aproximación al modelo. Recuperado julio 22 de 2010. Disponible en: www.researchgate. net/.../26633496_La_Gramtica_del_Papel_y_la_Referencia_una_aproximacin_al_modelo - Estados Unidos.

Guiraud, P. (1979). La gramática. Buenos Aires: Editorial Universitaria de Buenos Aires.

Halliday, M. A. K. (1982). El lenguaje como semiótica social. México: Fondo de Cultura Económica.
Hjelsmlev, L. (1976). Principios de gramática general. Madrid: Gredos.

Klinkenberg, J. (2006). Manual de Semiótica General. Bogotá: Fundación Universidad de Bogotá Jorge Tadeo Lozano.

López, A. (2000). Teoría gramatical. En: M. Álvar, M. (Director), Introducción a la lingüística española (7-22). Barcelona: Madrid.

Lorenzo, G. (2006). El vacío sexual, la tautología natural y la promesa minimalista. Madrid: Machado Libros.

Marcos, F. (1994). Introducción a la lingüística: historia y modelos. Madrid: Síntesis.

Mendívil, J. (2003). Gramática natural: La gramática generativa y la tercera cultura. Madrid: Machado Libros.

Santiago, A. (2007). La lengua: su estudio y su enseñanza. En Revista Praxis Pedagógica, (No. 8), 131-145.

Tobón, L. (2007). La lingüística del lenguaje. Estudios en torno a los procesos de significar y comunicar. Bogotá: UPN. 